\title{
Review on Offline Signature Recognition and Verification Techniques
}

\author{
Henali Patel \\ M.Sc. (IT), \\ Babu Madhav Institute of \\ Information Technology, \\ Bardoli, 394601
}

\author{
Shivani Desai \\ M.Sc. (IT), \\ Babu Madhav Institute of \\ Information \\ Technology, \\ Bardoli, 394601
}

\author{
Pooja Desai \\ M.Sc. (IT), \\ Babu Madhav Institute of \\ Information \\ Technology, \\ Bardoli, 394601
}

\author{
Abha Damani \\ Teaching Assistant \\ Babu Madhav Institute of \\ Information Technology, \\ Bardoli, 394601
}

\begin{abstract}
A mark or sign made by an individual on an instrument or document to signify knowledge, approval, acceptance, or obligation. Signature recognition is a behavioural biometric that identifies an individual on the basis of their handwritten text. In this paper we have given description about signature recognition methods and have compared all those methods.
\end{abstract}

\section{Keywords}

Signature Recognition, Pre-processing, OCR (Optical Character Recognition), Template Matching

\section{INTRODUCTION}

In earlier days, people verify their signature by comparing the signature with its sample which is already taken in one paper and at the time of verification they compare those two papers with each other. This technique is not so sufficient because it is time consuming and human may have error while detecting the signature so with the modern technique of signature recognition we can achieve a sufficient result.

Signature Recognition is a behavioural biometric. It can be operated in two different ways:

\subsection{Static}

In this mode, user write their signature on paper, digitize it through an optical scanner or camera, and the biometric system recognizes the signature analysing its shape.This group is also known as "Off-Line".

\subsection{Dynamic}

In this mode, users write their signature in digitizing tablet, which acquires the signature in real time.Another possibility is the acquisition by means of stylyus-operated PDAs. Some systems also operate on smart-phones or tablets with a capacitive screen, where users can use a finger or an appropriate pen. It is also known as "On-Line".Dynamic information usually consists of the following information:
- Spatial coordinate $\mathrm{x}(\mathrm{t})$

- Spatial coordinate $y(t)$

- $\quad$ Pressure $\mathrm{p}(\mathrm{t})$

- $\quad$ Azimuth az (t)

- Inclination $\mathrm{n}(\mathrm{t})$

- Pen up/down

There are a number of limitations in the data acquisition phase. The first is signature's length. In case of too long signatures the data analysis may be difficult for the recognition system to identify the unique data points. In addition, pre-processing and recognition process are time consuming. On the other hand, in case of too short signatures the data set may not be representative enough and false accept rate (FAR) coefficient may be too high (i.e. an impostor can be authorised by the system).

The second limitation is the environment and conditions where a person performs the enrolment and verification phase. For example, two signatures taken from an individual may substantially differ from each other only because the position of a person was different.

The proposed steps of signature recognition are as follow:

- Pre-Processing

- Feature Extraction

- Matching

- Verification

- Output 


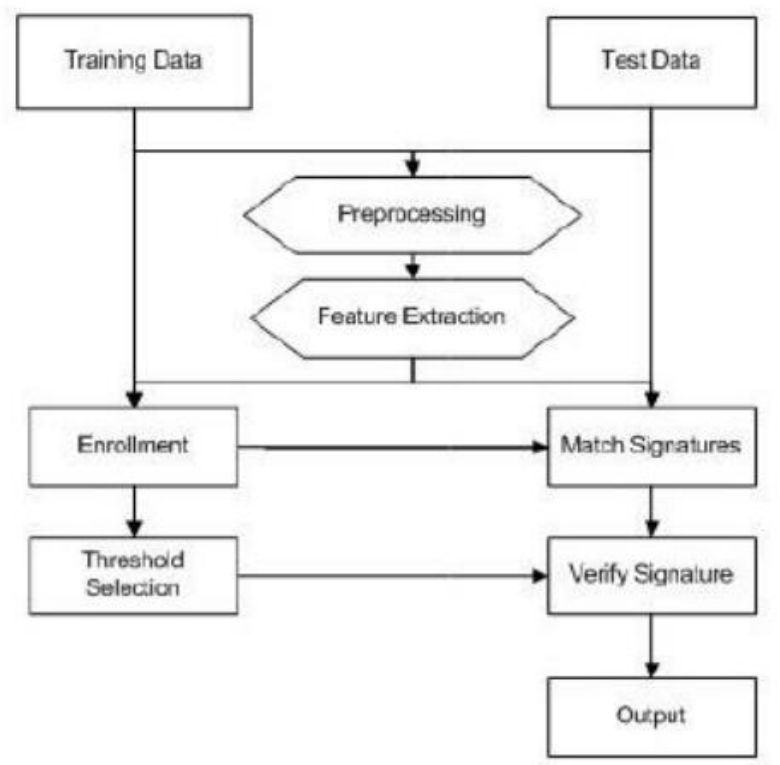

Fig [2.1]: Process of Signature Verification

\section{APPLICATION OF SIGNATURE RECOGNITION}

- Business

- Forensics Casework

- Banking

- Company

- School/colleges

\section{LITERATURE REVIEW}

D.Bertolinia, L.S. Oliveirab, *, E. Justinoa,R.Sabourinc[1] Reducing forgeries in writer-independent off-line signature verification through ensembleofclassifiers.in this paper they describe two important issues of off-line signature verification.

Luiz G. Hafemann1, Robert Sabourin1 and Luiz S. Oliveira2 [2] Offline Handwritten Signature Verification Literature Review. In this paper, they present how the problem has been handled in the past few decades, analyse the recent advancements in the field, and the potential directions for future research.

Cemil OZ, Sakarya University Computer Eng. Department, Sakarya, Turkey, Fikret Ercal, UMR Computer Science Department, Rolla, MO 65401, Zafer Demir, Sakaraya University electric electronic eng. Department sakarya, Turkey,[3] "Signature Recognition and Verification with ANN". In this paper, they present an off-line signature recognition and verification system which is based on moment invariant method and ANN.they use Artificial Neural Network for recognize the signature. Two separate ANNs are used; one for signature recognition and another for verification. Their recognition system exhibited a $100 \%$ success rate by identifying correctly all of the 30 signatures that it was trained for.
Shiwani Sthapak1, Minal Khopade2, Chetana Kashid3 [6] Artificial Neural Network Based Signature Recognition \& Verification. This paper deals with the off-line signature recognition \& verification using neural network in which the human signature is captured and presented in the image format to the system. Our recognition system exhibited 100\% success rate by identifying correctly all the signatures that it was trained for.

Ashwini Pansare, Shalini Bhatia [8] Off-line Signature Verification Using Neural Network. The method presented in this paper consists of image prepossessing, geometric feature extraction, neural network training with extracted features and verification. They explain error back propogation algorithm for signature recognition. Hence, the correct classification rate of the system is $85.7 \%$ in generalization.

Luiz G. Hafemann1, Robert Sabourin1 and Luiz S. Oliveira2[10] Offline Handwritten Signature Verification. This has demonstrated to be a challenging task, in particular in the offline (static) scenario that uses images of scanned signatures, where the dynamic information about the signing process is not available. They applied deep learning for signature recognition. In this paper, they present how the problem has been handled in the past few decades; analyze the recent advancements in the field, and the potential directions for future research.

Emre Özgündüz, Talen Şentürk and M. Elif Karsligil [12] offline signature verification and recognition system using the global, directional and grid features of signatures.in this paper they explain about Support Vector Machine (SVM) was used to verify and classify the signatures and a classification ratio of 0.95 was obtained.

Based on Literature Review the process of signature recognition is as follow: 


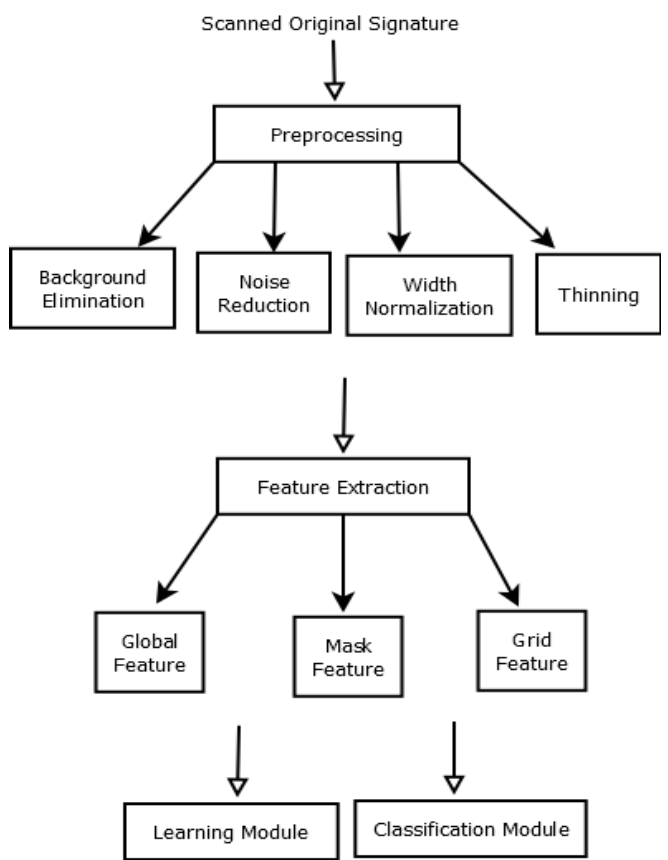

Fig [5.1]: Flow of Signature Recognition

\subsection{Preprocessing}

The pre-processing step is applied both in training and testing phases. Signatures are scanned in gray. The purpose in this phase is to make signatures standard and ready for feature extraction [12].

Signatures are scanned in gray, using following equations as [6]

Gray colour $=(0.299 *$ Red $)+(0.5879 *$ Green $)+(0.144 *$ Blue $)$

The pre-processing stage includes following steps: scaling, Background elimination, noise reduction, width normalization and skeletonization.

\subsubsection{Background Elimination}

Data area cropping must be done for extracting features. Ptile thresholding was chosen to capture signature from the background. After the thresholding the pixels of the signature would be "1" and the other pixels which belong to the background would be " 0 ".

The brightness threshold can be chosen such that it satisfies the following conditions: [6]

Suppose image pixels $f(x, y)$ then,

$$
\begin{aligned}
& \text { If }(x, y) \geq T \\
& \text { Then } f(x, y)=\text { Background } \\
& \text { Else } f(x, y)=\text { Object }
\end{aligned}
$$

\subsubsection{Noise Reduction:}

Images are contaminated due to stemming from decoding errors or noisy channels. An image also gets degraded because of the detrimental effects due to illumination and other objects in the environment. Median filter is extensively used for smoothing and restoring images corrupted by noise [8]. This is a nonlinear process useful principally in reducing impulsive noise [7]. In a median filter, a window slides over the image, and for each location of the window, the median concentration of the pixels within it decide the intensity of the pixel positioned in the middle of the window. As weigh against to the mean filter, median filter has striking properties for suppressing impulse noise while preserving edges; due to this feature we are recommended this filter in our proposed system [8].

\subsubsection{Scaling:}

Let $\mathrm{H}$ be the height of the inputted image \& $\mathrm{W}$ be the width of the inputted image [7]. We can fit the image uniform at $100 * 100$ pixels by using the following equation as,

$$
\text { Xnew }=(\text { Xold } * 100) / H ;
$$

Where Xnew \& Xold are calculated \& original X coordinate,

$$
\text { Ynew }=(\text { Ynew } * 100) / W ;
$$

Where Ynew \& Yold are calculated \& original Y coordinate. With these equations input image is transformed to uniformed $100 * 100$ pixels image [7].

\subsubsection{Width Normalization}

Signature dimensions may have intrapersonal and interpersonal differences. So, the image width is adjusted to a default value and the height will change without any change on height-to-width ratio. At the end of width normalization width dimension is adjusted to 100 .

Normalization process made use of the following equations:

Xnew $=[($ Xold-Xmin $) /(X \max -\mathrm{Xmin})] * \mathrm{M}$

Ynew $=[($ Yold-Ymin $) /($ Ymax-Ymin $)] * M$

Where, Xnew, Ynew = Pixel coordinates for the normalized signature,

Xold, Yold = Pixel coordinates for the original signature, $\mathrm{M}$ $=$ Width/height meant for the normalized signature

\subsubsection{Thinning}

The goal of thinning is to eliminate the thickness differences of pen by making the image one pixel thick. In this system Hilditch's Algorithm is used. 


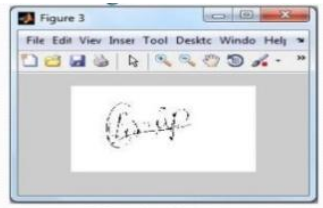

Binarized Image

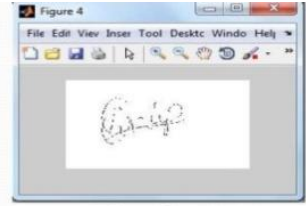

Thinned Image
Fig [5.1.1]: Image of Thinned Image.

\subsection{Feature Extraction}

Extracted features in this phase are the inputs of training phase. The features in this system are global features, mask features and grid features. Global features provide information about specific cases of the signature shape. Mask features provide information about directions of the lines of the signatures. Grid features provide overall signature appearance information.

\subsubsection{Global Features}

Signature area is the number of pixels which belong to the signature. This feature provides information about the signature density.

Signature height-to-width ratio is obtained by dividing signature height to signature width. Signature height and width can change. Height-to-width ratios of one person's signatures are approximately equal.

Maximum horizontal histogram and maximum vertical histogram: The horizontal histograms are calculated for each row and the row which has the highest value is taken as maximum horizontal histogram. The vertical histograms are calculated for each column and the column which has the highest value is taken as maximum vertical histogram. Horizontal and vertical center of the signature are calculated using the formulas in Eq. 1 [10].

\section{$Y \max X \max$}

\section{$\mathrm{Xmax} Y \max$}

Centrex $=\sum \mathrm{x} \sum \mathrm{b}[\mathrm{x}][\mathrm{y}]$

y $\sum b[\mathrm{x}][\mathrm{y}]$

Centery $=\sum$

$\mathrm{x}=1 \mathrm{y}=1, \mathrm{y}=1 \mathrm{x}=1$

$\mathrm{Ymax} X \max$

$$
\mathrm{Xmax} Y \max
$$

$$
\begin{aligned}
& \sum_{\mathrm{y}=1 \mathrm{x}=1} \sum_{\mathrm{x}=1 \mathrm{y}=1}^{\sum \mathrm{b}[\mathrm{x}][\mathrm{y}]} \\
&
\end{aligned}
$$

Local maxima numbers of the signature: The number of local maxima of the vertical and horizontal histogram is calculated.

Edge point numbers of the signature: Edge point is the pixel which has only one neighbour, which belongs to the signature, in 8-neighbor.

\section{Global Features}

- Height :

- Width :

- Number of Black Pixels :

- Centroid of the signature :

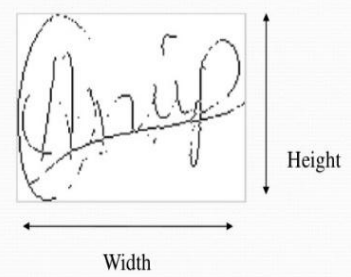

Fig [5.2.1]: Image of Global Features

\subsubsection{Mask Features}

Mask features provide information about directions of the lines of the signatures. The angles of the signatures have interpersonal differences. In this system 8 different $3 \times 3$ mask features are used [6]. Each mask is taken all around the signatures and the number of $3 \times 3$ parts of the signature, which are same with the mask, is calculated.

\subsubsection{Grid Features}

Grid features are used for finding densities of signature parts [10]. In this system 60 grid features are used. Signature is divided into 60 equal parts and the image area in each divided part is calculated.

\section{Grid Features}

- The cropped image is divided into 9 rectangular segments i.e.

(3 X 3 ) blocks.

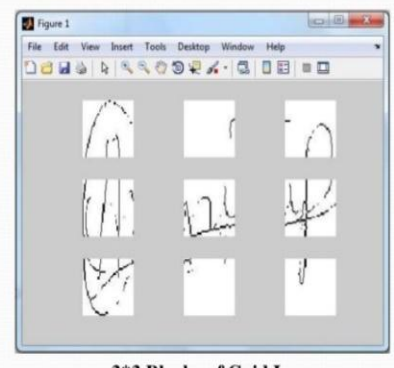

3*3 Blocks of Grid Image

Fig [5.2.2]: Image of Grid Features

\section{CLASSIFICATION METHODS 4.1 KNN Algorithm}

$\mathrm{K}$-nearest neighbour algorithm [12, 13] is a method for classifying objects based on closest training examples in the feature space. K-nearest neighbour algorithm is among the simplest of all machine learning algorithms. Training process for this algorithm only consists of storing feature vectors and labels of the training images. In the classification process, the unlabelled query point is simply assigned to the label of its $\mathrm{k}$ nearest neighbours

A main advantage of the $\mathrm{KNN}$ algorithm is that it performs well with multi-modal 2 classes because the basis of its decision is based on a small neighbourhood of similar objects. Therefore, even if the target class is multi-modal, the algorithm can still lead to good accuracy.

However a major disadvantage of the KNN algorithm is that it uses all the features equally in computing for similarities. This can lead to classification errors, especially when there is only a small subset of features that are useful for classification. 


\section{$4.2 \mathrm{NN}$}

An artificial neuron network (ANN) is a computational model based on the structure and functions of biological neural networks. Information that flows through the network affects the structure of the ANN because a neural network changes or learns, in a sense - based on that input and output.

ANNs are considered nonlinear statistical data modelling tools where the complex relationships between inputs and outputs are modelled or patterns are found.

ANN is also known as a neural network.

ANNs have three layers that are interconnected. The first layer consists of input neurons. Those neurons send data on to the second layer, which in turn sends the output neurons to the third layer.

Training an artificial neural network involves choosing from allowed models for which there are several associated algorithms.

\subsection{SVM}

Super Vector Machine (SVM) classification [14] uses different planes in space to divide data points using planes. An SVM model is a representation of the examples as points in space, mapped so that the examples of the separate categories or classes are divided by a dividing plane that maximizes the margin between different classes. This is due to the fact if the separating plane has the largest distance to the nearest training data points of any class, it lowers the generalization error of the overall classifier. The test points or query points are then mapped into that same space and predicted to belong to a category based on which side of the gap they fall on as shown in Figure 5.

A main advantage of SVM classification is that SVM performs well on datasets that have many attributes, even when there are only a few cases that are available for the training process. However, several disadvantages of SVM classification include limitations in speed and size during both training and testing phase of the algorithm and the selection of the kernel function parameters.

\subsection{Template Matching}

Template matching is the technique which used to finding small parts of an image which match template image. Template matching is widely used for processing images and pictures. In general, a technique includes its unique algorithm or method, which compares the template image with input image and finds similarity between them. It is the Best Way for Signature Recognition.

\subsection{FRR}

The false recognition rate, or FRR, is the measure of the likelihood that the biometric security system will incorrectly reject an access attempt by an authorized user. A system's FRR typically is stated as the ratio of the number of false recognitions divided by the number of identification attempts.

\subsection{Image Binarization}

Image Binarization is the process of separation of pixel values into dual collections, black as foreground and white as background. Thresholding has created to be a well-known technique used for Binarization of document images. Thresholding is further divide into the global and local Thresholding technique [19].

\subsubsection{Global Thresholding}

It compared each pixels gray level with a single global threshold.

\subsubsection{Local Thresholding}

Local thresholding means cropping the original image into 16 equal parts, once the cropping is done OTSU's algorithm is applied on all the cropped parts individually.

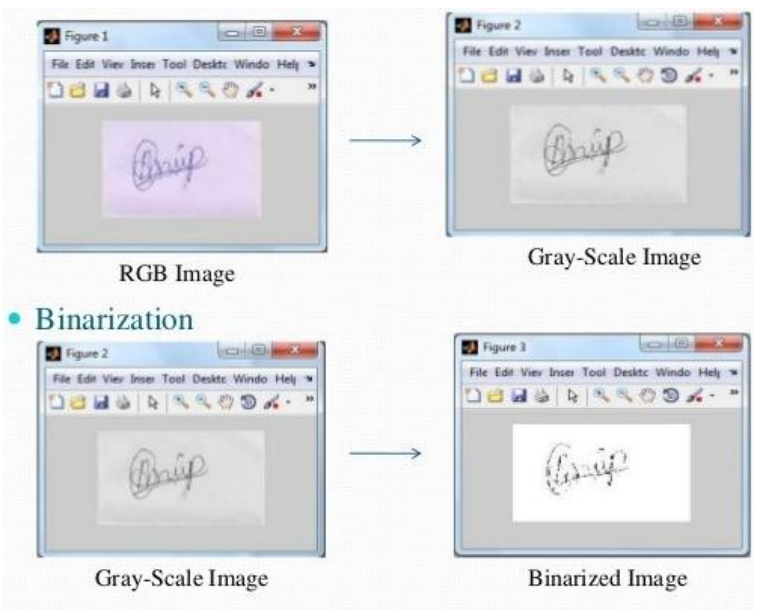

Fig [6.1]: Process of Binarization 


\section{COMPARISON OF METHOD}

\begin{tabular}{|c|c|c|c|c|}
\hline $\begin{array}{l}\text { Method } \\
\text { (Algorithm) }\end{array}$ & Dataset & Advantage & Disadvantage & Reference \\
\hline $\begin{array}{l}\text { KNN(Known } \\
\text { Nearest } \\
\text { Neighbour) }\end{array}$ & $\begin{array}{l}\text { Tobacco-800 } \\
\text { Dataset }\end{array}$ & $\begin{array}{l}\text {-Effective if the training data is } \\
\text { large }\end{array}$ & $\begin{array}{l}\text {-No clear idea about which type } \\
\text { Of distance to use } \\
\text { And which attribute } \\
\text { To use to produce } \\
\text { The best result. } \\
\text {-Computation cost } \\
\text { Is quite high. }\end{array}$ & [17] \\
\hline $\begin{array}{l}\text { NN(Neural } \\
\text { Network) }\end{array}$ & $\begin{array}{l}\text { Tobacco-800 } \\
\text { Dataset }\end{array}$ & $\begin{array}{l}\text {-Relatively } \\
\text { Easy to use. } \\
\text {-Great for complex } \\
\text { Problem like } \\
\text { Image recognition. }\end{array}$ & $\begin{array}{l}\text {-Require to shift } \\
\text { Load of training and } \\
\text { cases. } \\
\text {-Increasing accuracy by a few } \\
\text { percent can bump } \\
\text { up the scale by } \\
\text { several magnitudes. }\end{array}$ & {$[18]$} \\
\hline $\begin{array}{l}\text { SVM(Support } \\
\text { Vector machine) }\end{array}$ & $\begin{array}{l}\text { Tobacco-800 } \\
\text { Dataset }\end{array}$ & $\begin{array}{l}\text {-SVM performs } \\
\text { Well on data sets that have } \\
\text { many } \\
\text { attributes, even if there are very } \\
\text { few cases on which to train } \\
\text { the model. } \\
\text { Theres no upper } \\
\text { Limit on the } \\
\text { Number of attributes;the } \\
\text { Only constraints } \\
\text { are those } \\
\text { imposed by } \\
\text { hardware. }\end{array}$ & $\begin{array}{l}\text {-Bigest limitation } \\
\text { Of the support } \\
\text { Vector approach lies in choice of } \\
\text { the } \\
\text { kernel. } \\
\text {-The optimal design } \\
\text { for multiclass SVM } \\
\text { classifiers is a further area for } \\
\text { research. } \\
\text {-Another limitation } \\
\text { is speed and size, } \\
\text { both in training and } \\
\text { testing. }\end{array}$ & [19], [20] \\
\hline $\begin{array}{l}\text { OCR(Optical } \\
\text { Character } \\
\text { recognition }\end{array}$ & $\begin{array}{l}\text { Tobacco-800 } \\
\text { Dataset }\end{array}$ & $\begin{array}{l}\text {-Your document } \\
\text { can become } \\
\text { editable with } \\
\text { OCR.We can covert the files } \\
\text { to MS word } \\
\text { and any other } \\
\text { editable digital } \\
\text { formats. } \\
\text {-OCR allows you } \\
\text { to copy and paste from the } \\
\text { document itself whether that's in } \\
\text { PDF format or } \\
\text { MS word format. } \\
\text {-Saving you lots of time when } \\
\text { using a digital file rather than } \\
\text { paper documents. }\end{array}$ & $\begin{array}{l}\text {-Text from a source } \\
\text { with a font size of } \\
\text { less than } 12 \text { points } \\
\text { will results in more } \\
\text { enors. } \\
\text {-Most document formatting is lost } \\
\text { During text scanning, except for } \\
\text { Paragraph marks and tab stops. } \\
\text { Sometimes bold, italics and } \\
\text { underline are recognized, } \\
\text { depending on your software. } \\
\text {-Scanning of plain text files or } \\
\text { spreadsheet print } \\
\text { outs usually work, } \\
\text { however the data } \\
\text { needs to be reformatted to } \\
\text { match the original. }\end{array}$ & {$[21],[22]$} \\
\hline $\begin{array}{l}\text { Template } \\
\text { Matching }\end{array}$ & $\begin{array}{l}\text { Tobacco-800 } \\
\text { Dataset }\end{array}$ & $\begin{array}{l}\text { There is abundant physiological } \\
\text { support that simple features (lines } \\
\text { and edges of particular } \\
\text { orientations) are represented in } \\
\text { the nervous system with template- } \\
\text { like receptive fields in the visual } \\
\text { cortex. } \\
\text { They are amazingly reliable. If } \\
\text { the to-be encoded stimulus is } \\
\text { present, it's template will become } \\
\text { active. }\end{array}$ & $\begin{array}{l}\text { The difficulty with template } \\
\text { matching as a model for } \\
\text { perception is that contexts are } \\
\text { rarely constrained. } \\
\text { For instance, slight deviations in } \\
\text { shape, size, and orientation, } \\
\text { would prevent template matchers } \\
\text { from reading even the limited } \\
\text { number of letters (26) in English. } \\
\text { They are not inherently view } \\
\text { invariant. For every different } \\
\text { possible view, there would have } \\
\text { to be a different template } \\
\text { (replication). As such, template } \\
\text { representations are uneconomical. }\end{array}$ & {$[23]$} \\
\hline
\end{tabular}




\section{CONCLUSION}

Signatures are verified based on parameters extracted from the signature using various image processing techniques [11]. Our recognition system exhibited $100 \%$ success rate by identifying correctly all the signatures that it was trained for [8]. We did not consider this a "high risk" case because recognition step is always followed by verification step and these kinds of false positives can be easily caught by the verification system. Recognition and verification ability of the system can be increased by using additional features in the input data set. This study intends to reduce to a minimum the cases of forgery in business transactions [9].

\section{REFERENCES}

[1] D. Bertolini, L. S. Oliveira, E. Justino, and R. Sabourin. Reducing forgeries in writer-independent off-line signature verification through ensemble of classifiers. Pattern Recognition, 43(1), January 2010.

[2] R.K. Bharathi and B.H. Shekar. Off-line signature verification based on chain code histogram and Support Vector Machine. In 2013 International Conference on Advances in Computing, Communications and Informatics (ICACCI), pages 2063-2068, August 2013

[3] Alceu S. Britto, Robert Sabourin, and Luiz E. S. Oliveira. Dynamic selection of classifiers - a comprehensive review. Pattern Recognition, 47(11):3665-3680, November 2014.

[4] Rafael M. O. Cruz, Robert Sabourin, and George D. C. Cavalcanti. Dynamic classifier selection: Recent advances and perspectives. Information Fusion, 41:195216, May 2018.

[5] G.S. Eskander, R. Sabourin, and E. Granger. Hybrid writer-independentwriter-dependent offline signature verification system. IET Biometrics, 2(4):169-181, December 2013

[6] Shiwani Sthapak1, Minal Khopade2, Chetana Kashid3. Artificial Neural Network Based Signature Recognition \& Verification.ISSN 2250-2459, ISO 9001:2008 Certified Journal, Volume 3, Issue 8, and August 2013

[7] M.A. Ferrer, M. Diaz-Cabrera, and A. Morales. Synthetic off-line signature image generation. In 2013 International Conference on Biometrics (ICB), pages 1-7, June 2013.

[8] Ashwini Pansare, Shalini Bhatia "Off-line Signature Verification Using Neural Network", International Journal of Scientific \& Engineering Research, Volume 3, Issue 2, February-2012 1 ISSN 2229-5518

[9] S.T. Kolhe, S. E. Pawar, Dept. of Computer Engg, AVCOE, Sangamner, India, "Offline Signature Verification Using Neural Network", International Journal of Modern Engineering Research (IJMER), Vol.2, Issue.3, May-June 2012 pp-1171-1175

[10] Luiz G. Hafemann, Robert Sabourin, and Luiz S. Oliveira. Analyzing features learned for offline signature verification using Deep CNNs. In International Conference on Pattern Recognition, pages 2989-2994, 2016.

[11] Cemil OZ, Sakarya University Computer Eng. Department, Sakarya, Turkey, Fikret Ercal, UMR Computer Science Department, Rolla, MO 65401, Zafer Demir, Sakaraya University electric electronic eng. Department sakarya, Turkey, "Signature Recognition and Verification with ANN".

[12] Emre Özgündüz, Tülin Şentürk and M. Elif Karsligil OFF-LINE SIGNATURE VERIFICATION AND RECOGNITION BY SUPPORT VECTOR MACHINE.

[13] O.C Abikoye M.A Mabayoje R. Ajibade "Offline Signature Recognition \& Verification using Neural Network", Department of Computer Science University of Ilorin P.M.B 1515, Ilorin, Nigeria, International Journal of Computer Applications (0975 - 8887) Volume 35- No.2, December 2011

[14] Paigwar Shikha, Shukla Shailja, "Neural Network Based Offline Signature Recognition and Verification System", Department of Electrical Engineering, Jabalpur Engineering College Jabalpur, MP, INDIA, Research Journal of Engineering Sciences ISSN 2278 - 9472, Vol. 2(2), 11-15, February (2013)

[15] Yu Qiao, Jianzhuang Liu, Department of Information Engineering the Chinese University of Hong Kong, Xiaoou Tang, Microsoft Research Asia Beijing, China, "Offline Signature Verification Using Online Handwriting Registration".

[16] M. J. Alhaddad, D. Mohamad and A. M. Ahsan, "Online Signature Verification Using Probablistic Modeling and Neural Network", IEEE, (2012)

[17] http://people revoledu.com/kardi/tutorial/KNN/Strength\%20and\%20w eakness.htm

[18] https://www.quora.com/What-are-the-advantagesdisadvantages-of-Artificial-Neural-networks

[19] https://docs.oracle.com/cd/B28359_01/datamine.111/b28 129/algo_svm.htm\#CHHDJFDJ

[20] http://www.svms.org/disadvantages.html

[21] https://www.pearl-scan.co.uk/blog/advantages-of-acroptical-character-recognition

[22] http://www.meridianoutpost.com/resources/articals/ocrlimitations.php

[23] http://cmp.felk.cvut.cz/cmp/courses/dzo/resources/lecture _object-recognition.pdf.

[24] "A Review on the Various Techniques used for Optical Character Recognition", Pranob K Charles, V. Harish, M. Swathi, CH. Deepthi/International Journal of Engineering Research and Applications (IJERA) ISSN: 2248-9622, Vol. 2, Issue 1, Jan-Feb 2012. 